\title{
A Theoretical Study of the Supercontinuum Generation for the Photonic Crystal Fibre
}

\author{
Mohammed Salim Jasim ${ }^{1}$, Nadia Al-Aboody ${ }^{2}$ \\ \{msjadr72@gmail.com ${ }^{1}$, nadia.alaboody@stu.edu.iq $\left.{ }^{2}\right\}$
}

Ministry of Education, Missan Directorate of Education, Gifted Secondary School, Iraq ${ }^{1}$ Southern Technical University, Amara Technical Institute, Computer System Dep, Iraq ${ }^{2}$

\begin{abstract}
This work describes how numerical simulations based on the generalized nonlinear Schrödinger equation (GNLSE) have opened the way for a comprehensive physical understanding of the fundamental complex dynamics of supercontinuum generation (SCG) and Soliton fission (SC). SC was generated in optical fibres and fibre lasers that pumped by watt and kilowatt pulse sources, as well as in continuous-wave sources (CW) Light sources with increased spontaneous emission. An efficient approach for creating a continuous-wave source of soliton fission in the optical fibres pumped by $\mathrm{CW}$ light has been discovered. The ability to generate supercontinuum opens the door to a wide range of applications, from medicine to telecommunications.
\end{abstract}

Keywords: Photonic Crystal fibres (FCFs), Soliton, Generalized Nonlinear Schrödinger Equation (GNLSE), Supercontinuum generation (SCG), Continuous-Wave (CW).

\section{Introduction}

Photonic crystal fibre (PCF) technology has advanced steadily in recent years, attracting considerable interest from researchers [1-10]. In general, they are constructed of a single substance, such as silica, with tiny air holes running the length of their fibre. To obtain desirable optical features, such as indefinitely single-mode operation, several factors can be modified, including air hole arrangement, air hole pitch and diameter, air hole size, and the refractive index of the fibre material [5-11]. J. Wang et al. [12] proposed that PCFs be guided via a bandgap using elliptical air holes. H. Hassan [13] established two types of optical solid-core PCFs: total internal reflection and photonic bandgap. As a result, it appears that the suggested fibre is rather simple to produce. One of the most common uses of PCFs is supercontinuum generation (SCG) [14-16]. The spectral and temporal structure of a brief optical pulse changes as it passes through a Kerr nonlinearity and chromatic dispersion result in a transparent material. Apart from the invariant phase delay per unit propagation length, there are a few other factors to consider, the effects of dispersion and Kerr nonlinearity will perfectly cancel one another out, ensuring that even at long distances, the temporal and spectral structure of the pulses is retained [17]. While propagating in the nonlinear parameter $\mathrm{n}_{2}$ should be positive in a lossless medium, and the chromatic dispersion should be positive, and the pulse must have the same temporal form as an unchipped sech2 pulse. The generalized nonlinear Schrödinger (GNLSE) equation describes the propagation of pulses in a nonlinear index of refraction with a dispersive medium. The supercontinuum is created by nonlinearities operating on the ultra-shot as it passes through a ( PCF) with a highly nonlinear structure that separates into fundamental solitons, emitting 
dazzling white light. Induced Raman scattering allows the first-order solitons to migrate to the side of longer wavelengths as they pass through the fibre, resulting in a process known as induced Raman scattering (SSFS), with a much broader scope [18]. As shown in Fig 1 [19], the following behaviours indicate the mechanism of supercontinuum production via soliton fission based on the (GNLSE). To overcome the bandgap, this work proposes a spherical air hole with one solid core, emphasizing the importance of dimensions represented by the long wave.

The remaining of this paper is organized as follows: the next subsections include a detailed explanation for the soliton types, section II illustrates the achieved results, and section III demonstrates the conclusion of this work.

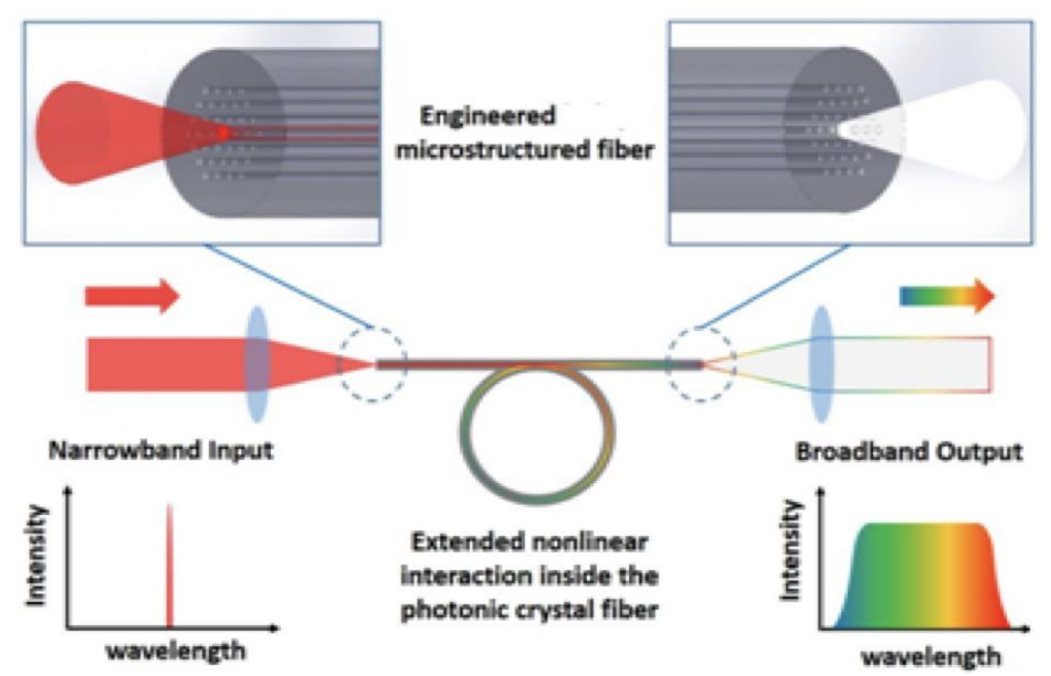

Fig 1. Supercontinuum generation.

\subsection{Optical Soliton}

Because of the medium's balance of dispersive and nonlinear effects, a soliton is an optical field that does not change during propagation. The two forms of optical solitons are spatial and temporal solitons [20-21]. When the electromagnetic field is spatially limited (as in optical fibres), temporal solitons develop, and the pulse form (in time) Because dispersion balances nonlinear effects, nothing changes. [22]. Nonlinear processes (self-focusing) balancing out diffraction would create spatial solitons [23-25] When the electromagnetic field is not locally restricted yet the form of the pulse in space does not change with the transmission. Fig 2 [19] illustrates this.

\subsection{Soliton fission}

Solitons are a fascinating representation of the nonlinear dynamics in nature that may be observed in a wide range of physics fields[24-25]. Solitons developing within optical fibres have gotten the most interest in the world of optics. With the development of new types of fibres, this fission mechanism has grown more relevant in recent years due to their nonlinear and dispersive properties, such as photonic-crystal fibres (PCF) [26]. 
Raman scattering, self-steepening, and cubic dispersion will all help to promote high-order soliton fission. These results, on the other hand, make it impossible to control the fission mechanism. Variation of chromatic dispersion between transmission lines (dispersion management) has been suggested as a method of regulating soliton dynamics [27-28]. Even when the spectrum of input pulses is just a few micrometres high, supercontinuum may be generated by ultrafast optical pulses traveling through such fibres (SC), and it can cover a broad spectral range from violet to infrared [29-30]. The fission of higher-order solitons is thought to be the fundamental physical mechanism causing SC creation in the anomalous-dispersion region [31].

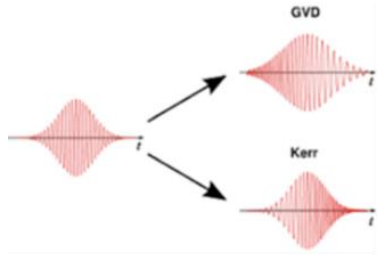

(a)

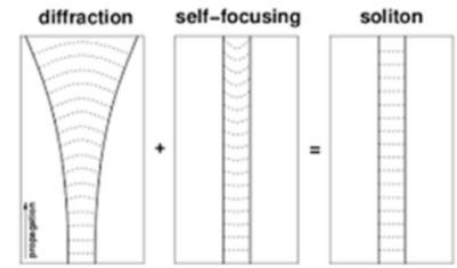

(b)

Fig 2. Linear and nonlinear effects on Gaussian pulses. (a) temporal soliton, (b) spatial soliton.

The generalized Schrödinger equation was used to achieve soliton fission and supercontinuum generation [32-33].

$$
\frac{\partial A}{\partial z}=-\frac{\alpha}{2}-\quad \sum_{m=2}^{M} i^{m-1} \frac{\beta_{m}}{m !} \frac{\partial^{m} A}{\partial t^{m}}+i\left(\gamma+i \gamma_{1} \frac{\partial}{\partial t}\right)\left(A(z, t) \int_{0}^{\infty} R\left(t^{\prime}\right)\left|A\left(z, t-t^{\prime}\right)^{2}\right| d t^{\prime}(1)\right.
$$

$\alpha$ represents the losses in the fibre since the duration of fibre used in supercontinuum generation is too short (only a few meters), these losses may be ignored in the computational simulation. $\mathrm{R}$ $\left(\mathrm{t}^{\prime}\right)$ is a nonlinearity function, which incorporates both electrical and nuclear components and makes use of the following formula.

$\left.R\left(t^{\prime}\right)=\left(1-f_{R}\right) \delta t-t_{e}\right)+f_{R} h_{R}(t)$

Because the electronic submission is treated as appearing instantly in this analysis, so $t_{e} \approx$ $1 \mathrm{fs}$. The Raman response feature, $\mathrm{h}_{\mathrm{R}}(\mathrm{t})$, provides details on the vibration of silica molecules. Since this is better determined experimentally, an empirical form of $h_{R}(t)$ exists (Blow and Wood, 1989), This is determined by the equation (3) [34].

$h_{R}(t)=\frac{\tau_{1}^{2}+\tau_{2}^{2}}{\tau_{1} \tau_{2}^{2}} e^{\left(\frac{-t}{\tau_{2}}\right)} \sin \left(\frac{t}{\tau_{1}}\right)$

Blow and Wood's(1989), experimental results indicate that $\mathrm{f}_{\mathrm{R}}=0.18, \tau_{1}=12.2 \mathrm{fs}$, and $\tau_{2}=32 \mathrm{fs}$, When the approximations $\alpha=0$ and $\tau_{1}=\frac{\gamma}{\omega_{0}}$ are added to equation (1), the following results are obtained:

$\frac{\partial A}{\partial z}=-\sum_{m=}^{M} 2 i^{m-1} \frac{\beta_{m}}{m !} \frac{\partial^{m} A}{\partial t^{m}}+i \gamma\left(1+\frac{i}{\omega \circ} \frac{\partial}{\partial t}\right)\left(A(z, t) \int_{0}^{\infty} R\left(t^{\prime}\right)\left|\mathrm{A}\left(z, t-t^{\prime}\right)^{2}\right| d t^{\prime}\right)$ 
Substituting equation (2) into the integral of equation (4) gives:

$$
\begin{aligned}
& \int_{0}^{\infty} R\left(t^{\prime}\right)\left|A\left(z, t-t^{\prime}\right)^{2}\right| d t^{\prime}=\left(1-f_{R}\right) \int_{0}^{\infty} \delta\left(t-t_{e}\right)\left|A\left(z, t-t^{\prime}\right)^{2}\right| \mathrm{d} t^{\prime}+ \\
& f_{R} \int_{0}^{\infty} h_{R}\left|A\left(z, t-t^{\prime}\right)^{2}\right| d t^{\prime} \\
& \quad \int_{0}^{\infty} R\left(t^{\prime}\right)\left|A\left(z, t-\square^{\prime}\right)^{2}\right| d t^{\prime}=\left(1-f_{R}\right)|A|^{2}+f_{R} \int_{0}^{\infty} h_{R}\left|A\left(z, t-t^{\prime}\right)^{2}\right| d t^{\prime}
\end{aligned}
$$

Therefore, equation (4) becomes:

$$
\begin{aligned}
& \frac{\partial A}{\partial z}=-\sum_{m-2}^{M} i^{m-1} \frac{\beta_{m}}{m !} \frac{\partial^{m} A}{\partial t^{m}}+i \gamma A\left(1+\frac{i}{\omega} \frac{\partial}{\partial t}\right)\left(\left(1-f_{R}\right)|A|^{2}+\right. \\
& \left.f_{R} \int_{0}^{\infty} h_{R}\left|A\left(z, t-t^{\prime}\right)^{2}\right| d t^{\prime}\right) \\
& \frac{\partial A}{\partial z}=-\sum_{m-1}^{M} i^{m-1} \frac{\beta_{m}}{m !} \frac{\partial^{m} A}{\partial t^{m}}+i \gamma\left(1-f_{R}\right)\left(A|A|^{2}+\frac{i}{\omega \circ} \frac{\partial}{\partial t}\left(A|A|^{2}\right)\right)+ \\
& i \gamma f_{R}\left(A \int_{0}^{\infty} h_{R}\left|A\left(z, t-t^{\prime}\right)^{2}\right| d t^{\prime}+\frac{i}{\omega \circ} \frac{\partial}{\partial t}\left[A \int_{0}^{\infty} h_{R}\left|A\left(z, t-t^{\prime}\right)^{2}\right| d t^{\prime}\right]\right)
\end{aligned}
$$

\subsubsection{Split step Fourier Method (STFM) for Solving the GNLSE}

Equation(5) can be solved using the SSFM. This method focuses on distinguishing between the equation's dispersive and nonlinear components. This means that over a limited distance, these pieces can be regarded as autonomous. As a result, we can write equation (5) as follows: [19]:

$\frac{\partial A}{\partial z}=(\widehat{D}+\widehat{N}) A$

Equations (7) and (8) give the dispersive term $\widehat{D}$ and the nonlinear terms $\widehat{N}$

$\widehat{D}=-\sum_{m=2}^{M} i^{m-1} \frac{\beta_{m}}{m !} \frac{\partial^{m}}{\partial t^{m}}$

and

$\widehat{N}=i \gamma\left(1-f_{R}\right)\left(|A|^{2}+\frac{i}{\omega \circ A} \frac{\partial}{\partial t}\left(A|A|^{2}\right)+i \gamma f_{R}\left(\int_{0}^{\infty} h_{R}\left|A\left(z, t-t^{\prime}\right)^{2}\right| d t^{\prime}+\right.\right.$ $\left.\frac{i}{\omega \circ A} \frac{\partial}{\partial t}\left[A \int_{0}^{\infty} h_{R}\left|A\left(z, t-t^{\prime}\right)^{2}\right| d t^{\prime}\right]\right)$

Equation (6) when solved yields

$A(z+h, t)=\exp [h(\widehat{D}+\widehat{N})] A(z, t)$

And

$\exp (h \widehat{D}) \exp (h \widehat{N})=\exp \left(h[\widehat{D}+\widehat{N}]+\frac{h^{2}}{2}[\widehat{D} \widehat{N}-\widehat{N} \widehat{D}]+\cdots\right)$ 
By disregarding the $\mathrm{h}^{2}$ and higher components of the exponential, the following description may be derived.

$\exp (h[\widehat{D}+\widehat{N}] \approx \exp (h \widehat{D}) \exp (h \widehat{N})$

As a result, the pulse envelope solution would be:

$A(z+h, t) \approx \exp (h \widehat{D}) \exp (h \widehat{N}) A(z, t)$

equation (12), was determined by

$A(z+h, t) \approx \exp \left(\frac{h}{2} \widehat{D}\right) \exp \left(\int_{z}^{z+h} \widehat{N}\left(z^{\prime}\right) d z^{\prime}\right) \exp \left(\frac{h}{2} \widehat{D}\right) A(z, t)$

Equation (13) would be accurate to around third order.

\section{The Achieved Numerical Results}

The results were obtained using MATLAB OdE45. Table 1 lists the model parameters utilized in this study.

Table 1. Parameters for simulation.

\begin{tabular}{ll}
\hline Parameters & \multicolumn{1}{c}{ Values } \\
\hline \multicolumn{1}{c}{ Photonic Parameters } \\
\hline Air hole diameter (d) & $1.4 \mu \mathrm{m}$ \\
Pitch $\left(^{\wedge}\right)$ & $1.6 \mu \mathrm{m}$ \\
Pitch length & $0.15 \mathrm{~m}$ \\
Number of holes & 6 \\
\hline $\begin{array}{l}\text { Wavelength } \\
\text { Zero Dispersion Wavelength } \\
\text { (ZDW) }\end{array}$ & $780 \mathrm{~nm}^{-1}$ \\
Nonlinearity $(\gamma)$ & $0.11 \mathrm{~W}^{-1} \mathrm{~m}^{-1}$ \\
$\begin{array}{l}\text { Fractional Raman } \\
\text { Contribution }\left(f_{r}\right)\end{array}$ & 0.18 \\
\hline
\end{tabular}


First, computational effects with standard experimental parameters and an anomalous GVD regime are presented using the complete GNLSE in equation (1), which was considered as in Table 1, depending on the global dispersion in Table 2.

Table 2. Coefficient values of the Taylor series for the PCF's GVD used in simulation, expansion at 835 nm.

\begin{tabular}{cccc}
\hline Coefficient & Value $\left(\frac{p s^{2}}{k m}\right)$ & Coefficient & Value $\left(\frac{p s^{2}}{k m}\right)$ \\
\hline$\beta_{2}$ & -11.830 & $\beta_{3}$ & $8.1038 \times 10^{-2}$ \\
$\beta_{4}$ & -9.5205 & $\beta_{5}$ & $2.0737 \times 10^{-7}$ \\
& $\times 10^{-5}$ & & \\
$\beta_{6}$ & -5.3943 & $\beta_{7}$ & $1.3486 \times 10^{-12}$ \\
& $\times 10^{-10}$ & & \\
$\beta_{8}$ & -2.5495 & $\beta_{9}$ & $3.0524 \times 10^{-18}$ \\
& $\times 10^{-15}$ & & \\
$\beta_{10}$ & -1.7140 & & \\
& $\times 10^{-21}$ & & \\
\hline
\end{tabular}

In the simulation, the initial infused pulse exhibits a hyperbolic secant field profile is $A(0, T)$, which is equal to $P_{0} \operatorname{sech}\left(\frac{T}{T 0}\right), T_{0}=28.4 \mathrm{fs}$ with $P_{0}=500 \mathrm{~W}$ input power and all other simulation results assume hyperbolic secant input pulses. The PCFs selection and the input pulse parameters selected were to enable us to explore the SCG process in conditions that are representative of numerous real-world scenarios.

The dynamics of the spectral and the temporal evolution are shown in the first set of results. This is investigated using the numerical solution provided in Eq. 5. Fig 3 represents the findings of PCF propagation across a $0.15 \mathrm{~m}$ length. 

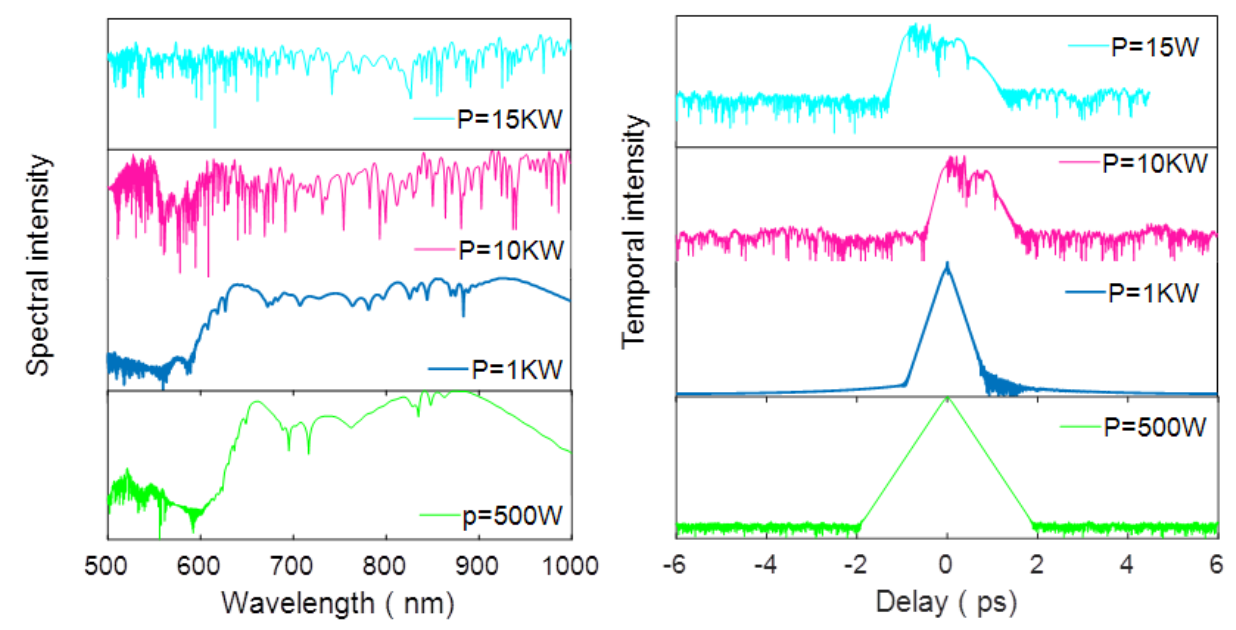

Fig 3. Supercontinuum generation across $0.15 \mathrm{~m}$ fibre.

Fig 3 shows the results of simulations at various power levels using an $835 \mathrm{~nm}$ pump wavelength. one, plot spectral and temporal slices as seen at representative propagation distances to demonstrate the fine structure of the resulting spectrum, and on a logarithmic scale, the SC spectra are displayed. Understanding the underlying physics, on the other hand, involves considering the dynamical progression of the initial pulse toward the output SC, which is a very complicated process. Moreover, the use of discrete layers has only captured a portion of the intricacy of these dynamics, and the density plot representation which is illustrated in Fig 4.

In this work, to describe both the spectral and the temporal intensity, the use of the logarithmic density scale is related to the highest value. The spectral broadening becomes asymmetric over this range, with discrete peaks of spectral emerging on both sides of the injected pump's long and short wavelengths, which are linked to the formation of separate peaks of temporal on a background with minimal amplitude. The logarithmic density chart shows the time backdrop. Although most spectral widening occurs early in the transmission process, subsequent phases are marked by a distinct continuous red-shift of the high-wavelength parts and temporal transform of distinct peaks in the time domain. The SCGs short wavelength edge does not expand in response to this constant shift to high wavelengths. On the other hand, the SCG's lowwavelength side does not propagate any further. All these features may be explained by describing the SCG manufacturing process physically. It is also self-evident that a simple and fundamental explanation for such complex dynamics, in terms of well-known characteristics of soliton transmission, The essential characteristics of the SCG development shown in the preceding figures may be easily understood. 

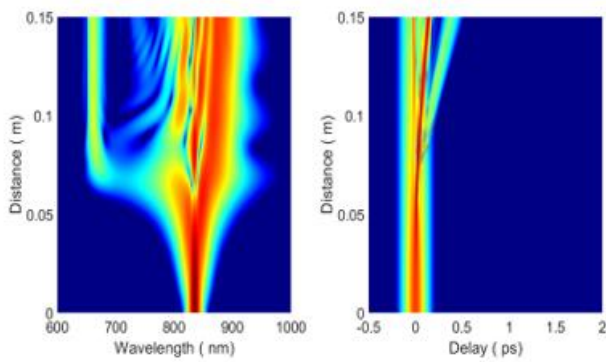

(a)
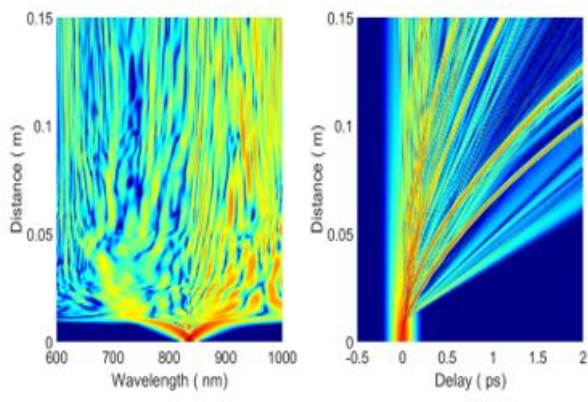

(c)
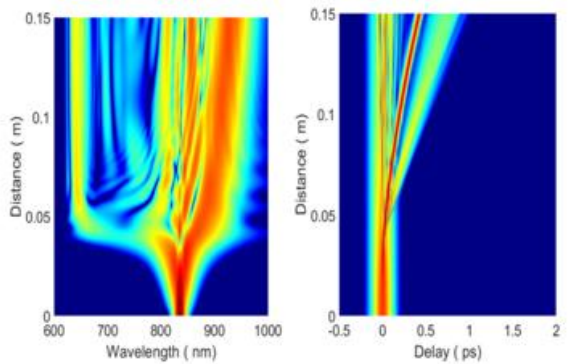

(b)
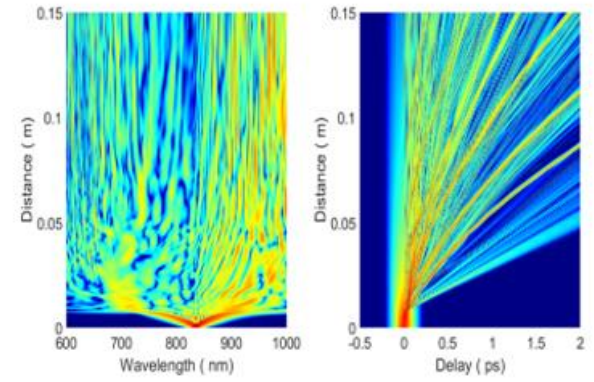

(d)

Fig 4. The density of the temporal and spectral development of SCG by ultra-short pulses with input powers of (a) $500 \mathrm{~W}$, (b) $1 \mathrm{KW}$, (c) $10 \mathrm{KW}$, and (d) $15 \mathrm{KW}$ are the results of numerical simulations. The input pulse wavelength is $850 \mathrm{~nm}$ and the FWHM duration is $50 \mathrm{fs}$.

In Fig 4, the soliton fission process dominates supercontinuum generation with increased input power pumping. Even though pulse breakup and decay signals may be seen at such a highpower level, at a lower power level, the fundamental physics may be illustrated more clearly. Raman scattering and Higher-order dispersion are the two most critical phenomena in the femtosecond range that might disturb such perfect periodic growth by causing pulse breakage owing to soliton fission. The input pulse power decides which of the two effects is dominant; however, the input pulse bandwidth is low enough for input pulses of 500W that the Raman perturbation frequently wins out. Furthermore, the pulse breakdown is generated by the dispersive disturbance for pulses with a power greater than $15 \mathrm{KW}$. Singular solitons are expelled from the input pulse one at a time in a methodical way, and the ejected solitons are sorted in ascending order by peak power, with the highest peak power at the top. The point at which fission begins is usually the same as when the injected higher power reaches its maximum value The temporal and spectral properties are very complex, as shown in figure 4 , the spectrum exhibits substantial modulation behaviour across short distances, although this can be explained simply as the result of spectral interference between several solitons on a linear scale, whose bandwidths continue to converge at this moment.

\section{Conclusion}

Supercontinuum creation is a complicated procedure, and any measurable description in terms of the basic physics, regardless of application, must take into consideration a variety of 
fibre and pulse characteristics. Understanding the physics behind the SCG creation process is frequently more essential than knowing how to efficiently select a pump source and fibre type to produce an SCG with certain desired characteristics.

Looking into the SC generation in fibre lasers and photonic crystal fibres pumped by various power levels, ranging from $500 \mathrm{~W}$ to $15 \mathrm{KW}$. A ring laser with nanostructure nonlinear fibre shows SC production within a $650-950 \mathrm{~nm}$ spectral bandwidth of $500 \mathrm{~W}$. While the bandwidth grew to more than $600-1000 \mathrm{~nm}$ at $15 \mathrm{KW}$. Also, this study suggested and demonstrated a novel design method for producing a CW SC in an optical fibre pumped by CW light. This type of spectral expansion has more important in many modern applications including medical, industrial, and military, and play an important role in communication systems.

\section{References}

[1] Knight JC, Birks TA, Russell PS, Atkin DM. All-silica single-mode optical fibre with photonic crystal cladding: errata. Optics Letters. 1997 Apr 1;22(7):484-5.

[2] Russell P. Photonic crystal fibres. science. 2003 Jan 17;299(5605):358-62.

[3] Chiang JS, Wu TL. Analysis of propagation characteristics for an octagonal photonic crystal fibre (O-PCF). Optics Communications. 2006 Feb 15;258(2):170-6.

[4] Akowuah EK, Gorman T, Ademgil H, Haxha S, Robinson GK, Oliver JV. Numerical analysis of a photonic crystal fibre for biosensing applications. IEEE Journal of Quantum Electronics. 2012 Aug 17;48(11):1403-10.

[5] Hossain M, Maniruzzaman M. Prospect of Photonic Crystal Fibre (PCF) In Medical Science. InProceedings of 4th Global Engineering, Science and Technology Conference, BIAM Foundation, Dhaka, Bangladesh 2013 (pp. 1-5).

[6] Dash JN, Jha R. Graphene-based birefringent photonic crystal fibre sensor using surface plasmon resonance. IEEE Photonics Technology Letters. 2014 Apr 7;26(11):1092-5.

[7] Morshed M, Arif MF, Asaduzzaman S, Ahmed K. Design and characterization of photonic crystal fibre for sensing applications. European Scientific Journal. 2015 Apr 1;11(12).

[8] Kumar P, Fiaboe KF, Roy JS. Highly birefringent do-octagonal photonic crystal fibres with ultra flattened zero dispersion for supercontinuum generation. Journal of Microwaves, Optoelectronics and Electromagnetic Applications. 2019 Mar;18(1):80-95.

[9] Agbemabiese PA, Akowuah EK. Numerical analysis of photonic crystal fibre of ultra-high birefringence and high nonlinearity. Scientific Reports. 2020 Dec 3;10(1):1-2.

[10] Van Le H, Nguyen HT, Buczynski R, Kasztelanic R. Supercontinuum generation in photonic crystal fibres infiltrated with tetrachloroethylene. Optical and Quantum Electronics. 2021 Apr;53(4):1-8.

[11] Graydon O. Single-mode operation. Nature Photonics. 2018 Aug;12(8):443-.

[12] Wang J, Jiang C, Hu W, Gao M. High birefringence photonic bandgap fibre with elliptical air holes. Optical Fibre Technology. 2006 Jul 1;12(3):265-7.

[13] Hassan HF. Studying Enhancement of Humidity Sensor Based on Photonic Crystal Fibre Interferometer (Doctoral dissertation, University of Baghdad).

[14] Sharafali A, Nithyanandan K. A theoretical study on the supercontinuum generation in a novel suspended liquid core photonic crystal fibre. Applied Physics B. 2020 Apr;126(4):1-2.

[15] Le Canh T, Le Van H, Pysz D, Dinh TB, Nguyen DT, Dinh QH, Klimczak M, Kasztelanic R, Pniewski J, Buczynski R, Dinh KX. Supercontinuum generation in all-normal dispersion suspended core fibre infiltrated with water. Optical Materials Express. 2020 Jul 1;10(7):1733-48.

[16] Kumar P, Fiaboe KF, Roy JS. Design of nonlinear photonic crystal fibres with ultra-flattened zero dispersion for supercontinuum generation. ETRI Journal. 2020 Apr;42(2):282-91.

[17] Zhao S, Sun X. Soliton dynamics in an all-normal-dispersion photonic crystal fibre with frequencydependent Kerr nonlinearity. Physical Review A. 2020 Sep 16;102(3):033514. 
[18] Scheibinger R, Lüpken NM, Chemnitz M, Schaarschmidt K, Kobelke J, Fallnich C, Schmidt MA. Higher-order mode supercontinuum generation in dispersion-engineered liquid-core fibres. Scientific reports. 2021 Mar 5;11(1):1-1.

[19] Mohammed Salim Jasim, Soliton and Supercontinuum Generation in Photonic Crystal Fibres, PhD. thesis, University of Basrah,Iraq. 2019

[20] López-Zubieta BA, Jarque EC, Sola ÍJ, San Roman J. Spatiotemporal-dressed optical solitons in hollow-core capillaries. OSA Continuum. 2018 Nov 15;1(3):930-8.

[21] Al Qarni AA, Alshaery AA, Bakodah HO. Optical solitons for the Lakshmanan-Porsezian-Daniel model by collective variable method. Results in Optics. 2020 Nov 1;1:100017.

[22] Englebert N, Arabí CM, Parra-Rivas P, Gorza SP, Leo F. Temporal solitons in a coherently driven active resonator. Nature Photonics. 2021 May 10:1-6.

[23] Tiofack CG, Ndzana FI, Mohamadou A, Kofané TC. Spatial solitons and stability in the onedimensional and the two-dimensional generalized nonlinear Schrödinger equation with fourth-order diffraction and parity-time-symmetric potentials. Physical Review E. 2018 Mar 9;97(3):032204.

[24] Bagri M, Kumar S. Solitons transmission system: A dynamic shift in optical fibre communication. Indian Jour-nal of Science and Technology. 2020 Aug 19;13(30):2193-202.

[25] Blair SM. Optical soliton-based logic gates. University of Colorado at Boulder; 1998.

[26] Golovchenko EA, Dianov EM, Prokhorov AM, Serkin V. Decay of optical solitons. ZhETF Pisma Redaktsiiu. 1985 Jul;42:74.

[27] Tai K, Hasegawa A, Bekki N. Fission of optical solitons induced by stimulated Raman effect. Optics letters. 1988 May 1;13(5):392-4.

[28] Wai PK, Menyuk CR, Lee YC, Chen HH. Nonlinear pulse propagation in the neighborhood of the zero-dispersion wavelength of monomode optical fibres. Optics letters. 1986 Jul 1;11(7):464-6.

[29] Genty G, Lehtonen M, Ludvigsen H. Effect of cross-phase modulation on supercontinuum generated in microstructured fibres with sub-30 fs pulses. Optics express. 2004 Sep 20;12(19):4614-24.

[30] Adamu AI, Habib MS, Smith CR, Lopez JE, Jepsen PU, Amezcua-Correa R, Bang O, Markos C. Noise and spectral stability of deep-UV gas-filled fibre-based supercontinuum sources driven by ultrafast mid-IR pulses. Scientific reports. 2020 Mar 18;10(1):1-0.

[31] Zhou R, Huang R, Li Q, Fu HY. Raman soliton at $2 \mu \mathrm{m}$ in picosecond pumped supercontinuum by a weak CW trigger. Optics express. 2019 Apr 29;27(9):12976-86.

[32] Dudley JM, Genty G, Coen S. Supercontinuum generation in photonic crystal fibre. Reviews of modern physics. 2006 Oct 4;78(4):1135.

[33] Travers JC. Blue extension of optical fibre supercontinuum generation. Journal of Optics. 2010 Oct 29;12(11):113001.

[34] Blow KJ, Wood D. Theoretical description of transient stimulated Raman scattering in optical fibres. IEEE Journal of Quantum Electronics. 1989 Dec;25(12):2665-73. 